\title{
A Fiber Optic Sensor for the Measurement of Surface Roughness and Displacement Using Artificial Neural Networks
}

\author{
Kuiwei Zhang, Clive Butler, Qingping Yang, Member, IEEE, and Yicheng Lu
}

\begin{abstract}
This paper presents a fiber optic sensor system. Artificial neural networks (fast back-propagation) are employed for the data processing. The use of the neural networks makes it possible for the sensor to be used both for surface roughness and displacement measurement at the same time. The results indicate $100 \%$ correct surface classification for ten different surfaces (different materials, different manufacturing methods, and different surface roughnesses) and displacement errors less then $\pm \mathbf{5} \mu \mathrm{m}$. The actual accuracy was restricted by the calibration machine. A measuring range of $\pm 0.8 \mathrm{~mm}$ for the displacement measurement was achieved.
\end{abstract}

Index Terms - Artificial neural network, displacement, fiber optic sensor, measurement, surface roughness.

\section{INTRODUCTION}

$\mathbf{S}^{-}$ URFACE roughness and displacement are two very important characteristics for the products of manufacturing industry. Significant work has already been done in the measurement of surface roughness and displacement. The classical methods for these measurements are usually based on mechanical contact. Over the past decade, optical methods for measuring surface roughness and displacement have been used in industrial applications [1], [2]. Fiber optic sensors can also be fund of use in surface roughness and displacement measurement. With the characteristics of small size, high speed of response, great accuracy, good reliability, freedom from electromagnetic interference, and most important-nondestructiveness, the fiber optic sensor is increasingly used in industry for manufacturing process monitoring and automation [3]-[6].

\section{A Fiber Optic Sensor System}

This paper introduces a fiber optic sensor system, which is shown in Fig. 1. This system includes a light source, a $Y$-coupler, a fiber optic sensor, photodetectors, an amplifier circuit, a simultaneous sampling and hold circuit, an A/D card, and a computer.

A fiber optic sensor has been developed, shown in Fig. 2. It uses the reflective principle and the scattering properties of

Manuscript received June 3, 1996.

The authors are with Brunel University, Uxbridge, Middlesex UB8 3PH, U.K. (e-mail: empgkkz@brunel.ac.uk).

Publisher Item Identifier S 0018-9456(97)06473-5.

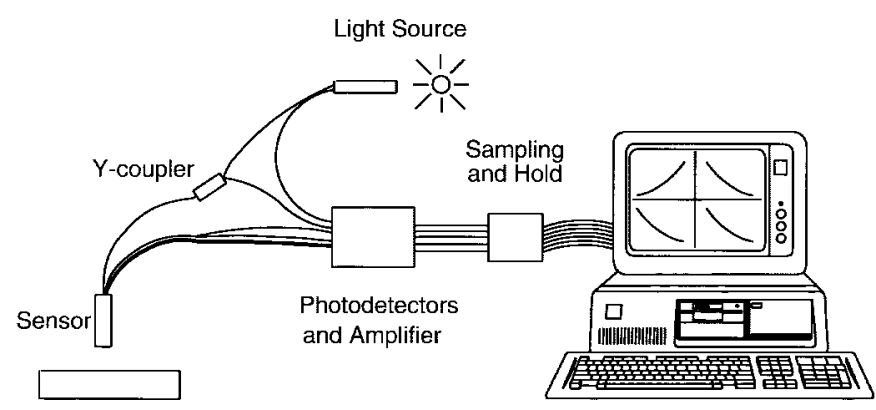

Workpiece

Fig. 1. Fiber optic sensor system.

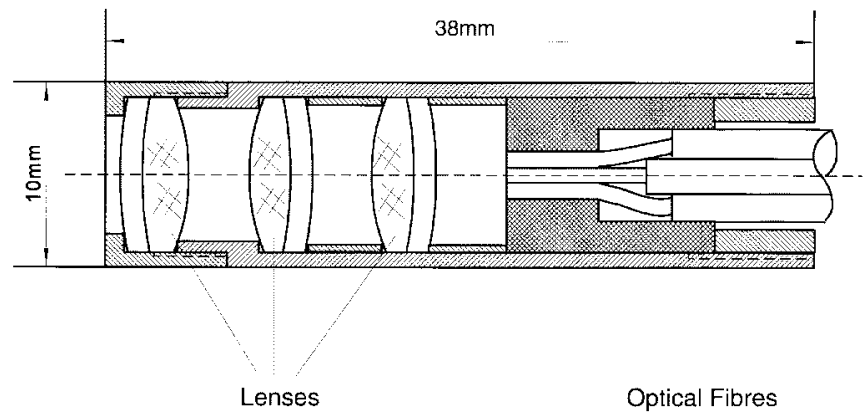

Fig. 2. Sensor for surface roughness and displacement.

light. A bundle of fibers is used for collecting the reflected and scattered light. The center fiber is used both for emitting and collecting the light. The use of lenses makes the stand off distance much larger and this is very important for on-line use of the fiber optic sensor. One of the lenses is designed to be changeable so that different standoff distances and different measuring ranges can be achieved. Compared with existing sensors, it is very small (length: $38 \mathrm{~mm}$, diameter: $10 \mathrm{~mm}$ ) and could be even smaller. This is comparable in size to conventional LVDT contact probes.

A new kind of $Y$-coupler is also introduced. This very simple coupler makes it possible for the center fiber to be used both for emitting and collecting the light. The light source is monitored during the measuring process and the influence of the change of the light source on the output of the sensor can be compensated. A simultaneous sample and hold circuit is introduced to get the signals at the same time for the same 


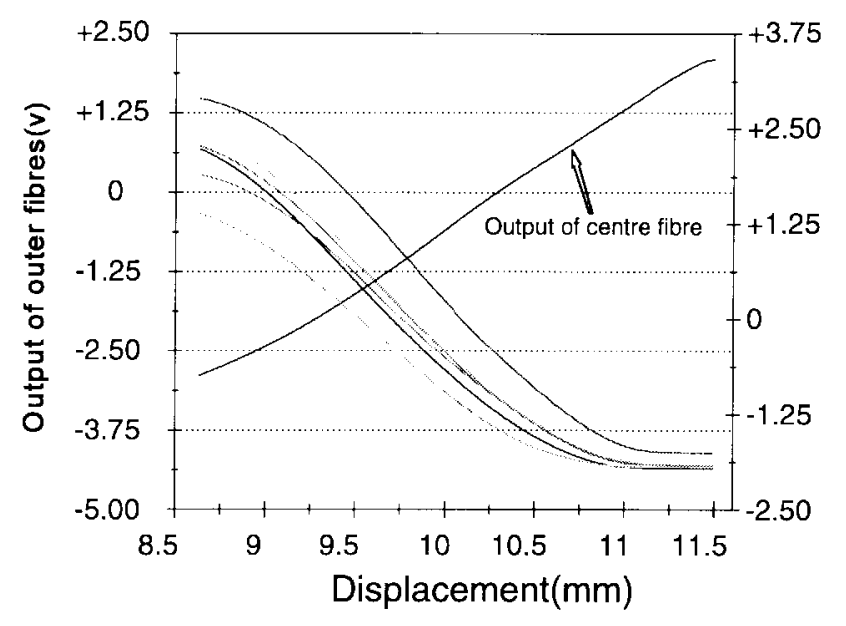

Fig. 3. Sensor output for coarse ground surface.

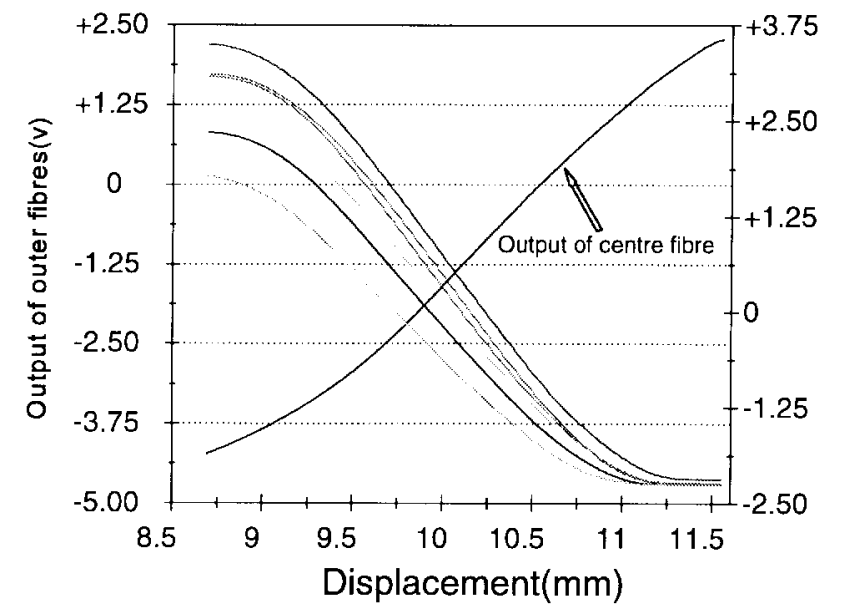

Fig. 4. Sensor output for fine ground surface.

displacement. This makes the results of the measurement more accurate.

\section{MEASURING PRINCIPLE}

The light from the center fiber is emitted on to the measuring surface and then reflected and scattered back to the fiber bundle. Generally speaking, the signals (proportional to the collected light intensities) depend mainly on the displacement and the surface roughness (including finish, texture, and reflectivity). The closer the surface departs from the focused point, the more light is collected by the center fiber and the less light collected by the outer fiber. The rougher the surface is, the less light is specularly reflected and the more the light scattered. The following function can be used to describe the variation of the light intensities with the displacement and the surface roughness

$$
\mathbf{I}=f(\mathbf{d}, \mathbf{r}) \text {. }
$$

Where $\mathbf{I}$ is the light intensity vector; $\mathbf{d}$ is the displacement and $\mathbf{r}$ is the surface roughness. The outputs of sensor for coarse ground surface and for fine ground surface are shown in Figs. 3 and 4. From these two figures we can see clearly the actual relationships.

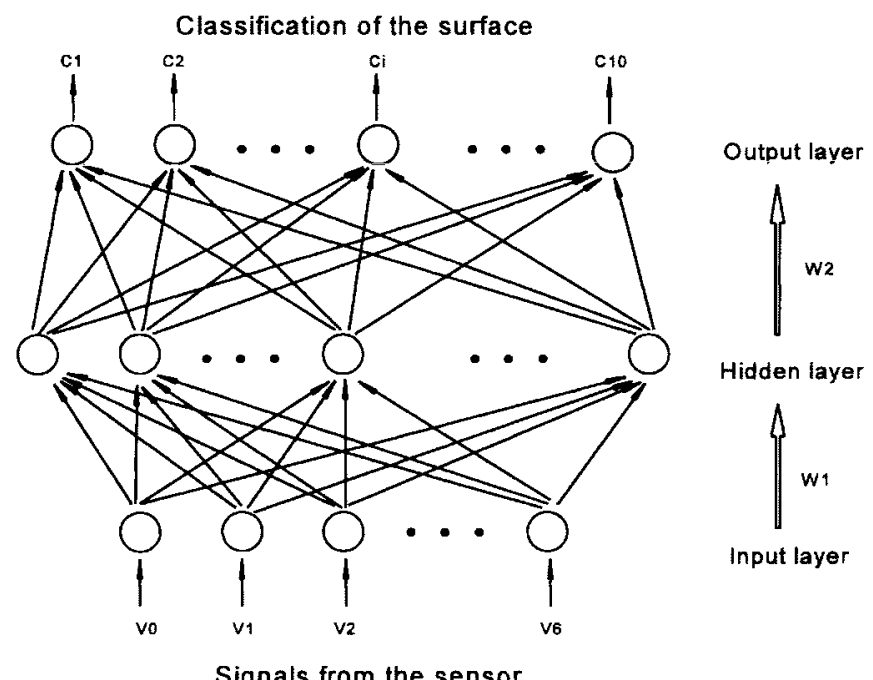

Fig. 5. Neural network for surface classification.

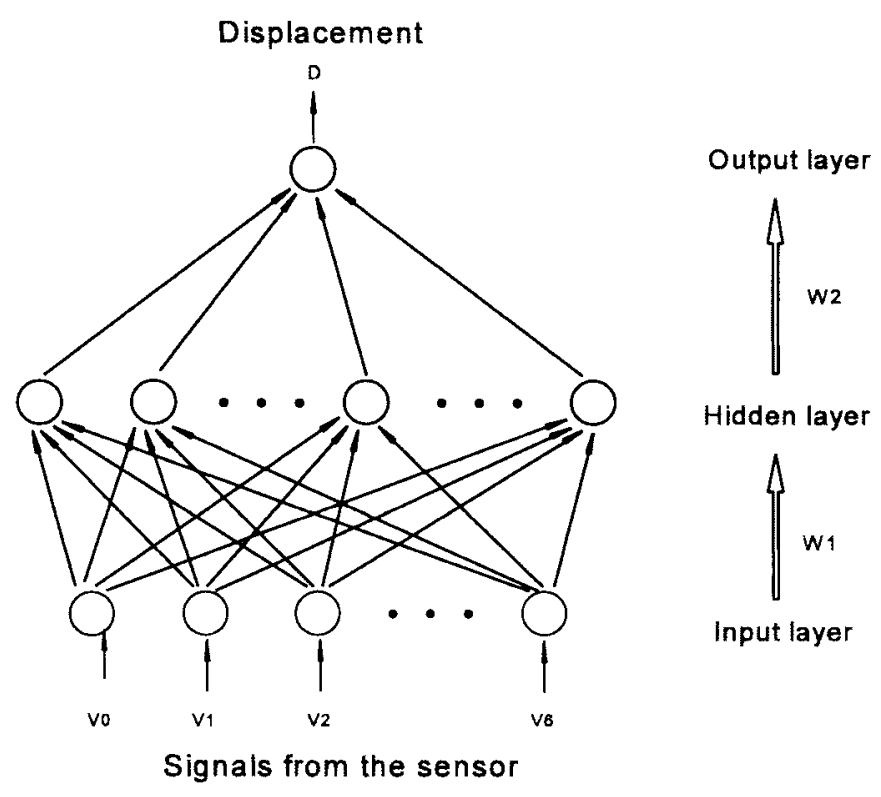

Fig. 6. Neural network for displacement.

\section{EXPERIMENT AND DATA PROCESSING}

The experiment involves the calibration of the sensor against ten different samples, including polished, milled (steel), coarse ground, fine ground, spark eroded, matt black (painted), matt white (painted), milled (aluminum), perspex, and nylon surfaces. There are big variations among the outputs of the sensor from the different surfaces. The gains and the offsets of the amplifier circuit is designed to be adjustable. The workpieces are placed on the motor driven table. The displacement and the outputs of the sensor are recorded simultaneously. The outputs of the sensor are voltages, which are represented by vector $\mathbf{V}$.

The function described in Section III is only qualitative, not a quantitative description. Finding the quantitative relationship between the light intensity and the displacement and between the light intensity and the surface roughness is not an easy job. An artificial neural network is the most appropriate technique to solve this kind of problem [7]-[9]. 


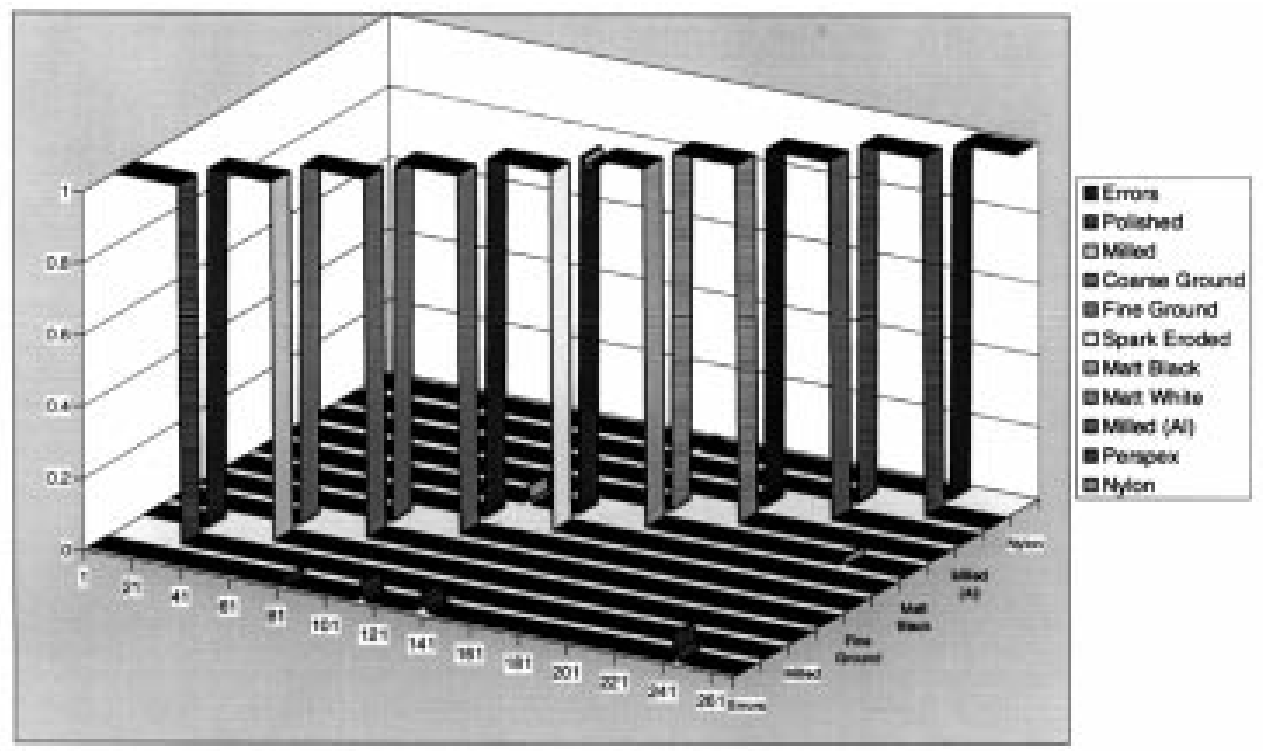

Fig. 7. Classification for surfaces.

In order to determine the displacement and the surface roughness from the signals derived from the collected light intensities, two separate artificial neural networks (fast backpropagation) are used for data processing. The artificial neural network A (ANN-A), shown in Fig. 5, is for surface classification, and the artificial neural network B (ANN-B), shown in Fig. 6, is for displacement.

The outputs of sensor $\mathbf{V}$, which are scaled to $(-1,+1)$ to cope with the input function, and the code $n$ ( $n=$ $1,2, \cdots, 10)$ of the samples are used to train the network ANN-A. ANN-A is a seven-input and ten-outputs three-layers network. The input activation function is set to $\tanh (D \cdot x)$ and the output activation function is set to linear function, $D \cdot x$. The range for input is $(-1,+1)$. The output is a surface code in digital form. For example, $\left[\begin{array}{lllll}1 & 0 & 0 & \cdots & 0\end{array}\right]$ represents the no. 1 (polished) surface, $\left[\begin{array}{lllll}0 & 0 & \cdots & 0 & 1\end{array}\right]$ represents the no. 10 (nylon) surface, and so on. The number of hidden cells is difficult to optimize. It is chosen mainly based on experience. Twelve hidden cells are used for this sample.

The same outputs of sensor $\mathbf{V}$ and the displacement $\mathbf{d}$ are used to train the network ANN-B. ANN-B is a seven-input and one-output three-layers network. The input activation function is set to $\tanh (D \cdot x)$ and the output activation function is set to linear function, $D \cdot x$. The ranges for input and output both are $(-1,+1)$. The output of displacement is in continuous analog form. The number of hidden cells is ten in this sample. Actually, ANN-B are trained ten times using the ten different sets of data from ten different samples.

The initial seed values for the neural networks influence the time of training. Several initial seed values have been tried. The learning rate is very important for the overall training speed. If it is too small, convergence will be very slow. If it is too large, convergence initially will be very fast, but the algorithm will eventually oscillate and thus not reach a minimum. $1 / N$ or $2 / N$ is recommended for the learning rate. $N$ is the number of the training items.
Sometimes a network may get itself trapped in local minima with no way out. In this case, "kicking" the network is necessary. This is to increase or decrease all the weights in the certain range by a random number.

\section{Measuring Results AND ANAlysis OF ERrors}

After the training of the neural network, the weights of the neural network can be saved. They can be reloaded at any time to test the experiment data to determine the results. The results for the surface classifications are shown in Fig. 7. The data are from the same ten surfaces but the different collections. The errors depend on the confidence level. The confidence level in this figure is $95 \%$.

The results for the displacement and errors are shown in Fig. 8. This is an example (coarse ground surface) for the displacement. The errors are the differences between the output of the neural network and the displacement measured by the calibration machine (profile projector). The output and the displacement are nearly on the same line. The difference is shown on a different scale to illustrate the errors. The errors depend on the measuring range and also the confidence level. The confidence level in this figure is $99 \%$.

The measuring results indicate that $100 \%$ correct surface classification for ten different surfaces and errors less then $\pm 5 \mu \mathrm{m}$ (within the measuring range of $\pm 0.8 \mathrm{~mm}$ ) for the displacement measurements. The result would be significantly improved if a more precise reference displacement measuring instrument had been available.

In order to get more accurate results some improvements have been made. These include the monitoring of the light source; a simultaneous sampling and hold circuit for the A/D converter, and some data processing techniques for the data collection. Ambient temperature is another important parameter that influences the results. Compensation would provide for further improvement. Different kinds of fibers have 


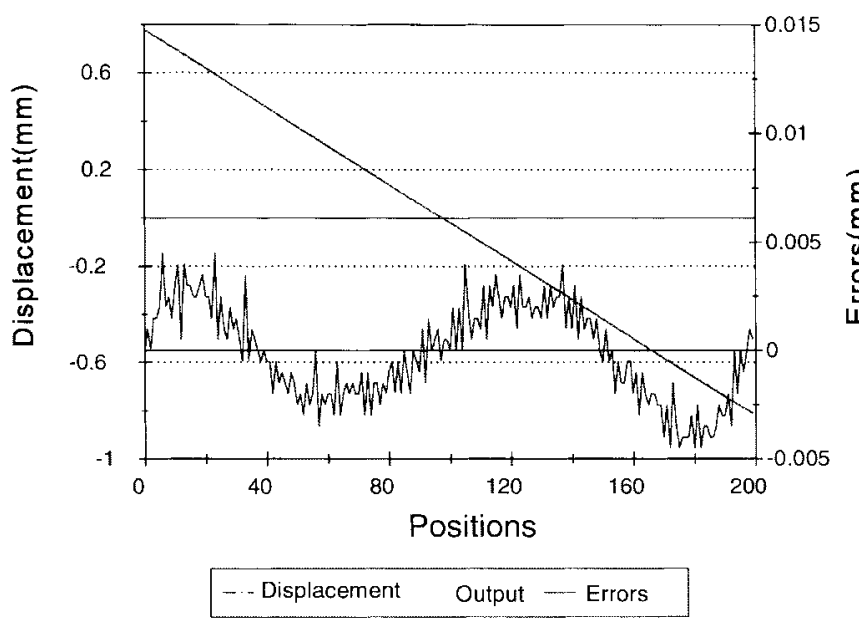

Fig. 8. Displacement and errors.

also been tried. Parameters such as the materials, the diameter and the numerical aperture of fibers all have an influence on the diameter of the focal spot and also on the precision of the measurements.

\section{CONCLUSIONS}

The sensor introduced in this paper is very compact and the use of a neural network is very effective in analyzing the data.

The sensor can be used for on-line monitoring during the manufacturing process for both surface roughness and displacement. Soft deformable materials can also be measured. It will find applications in automatic inspection and for quality control in manufacturing industry.

The results indicate that $100 \%$ correct surface classification for ten different surfaces (different materials, different manufacturing methods, and different surface roughnesses) and errors less then $\pm 5 \mu \mathrm{m}$ (actual accuracy was restricted by the calibration machine) with a measuring range of $\pm 0.8 \mathrm{~mm}$ for the displacement measurements.

\section{REFERENCES}

[1] R. Brodmann, G. Rodenstock, and G. Thurn, "An optical instrument for measuring the surface roughness in production control," Ann. CIRP, vol. 33, no. 1, p. 403, 1984

[2] K. Yamazaki, K. S. Lee, H. Aoyama, and M. Sawabe, "Noncontact probe for continuous measurement of surface inclination and position using dynamic irradiation of light beam," Ann. CIRP, vol. 42, no. 1, p. $585,1993$.

[3] P. J. Murphy and T. P. Coursolle, "Fiber optic displacement sensor employing graded index lens," Appl. Opt., vol. 29, no. 4, p. 544, 1990

[4] M. Abe, S. Ohta, and M. Sawabe, "Surface inspection using optical fiber sensor," in Proc. SPIE, 1990, vol. 1332, p. 366.

[5] A. W. Domanski and T. R. Wolinski, "Surface roughness measurement with optical fibers," IEEE Trans. Instrum. Meas., vol. 41, p. 1057, Dec. 1992.

[6] T. Pfeifer, "Fiberoptics for in-line production measurement," Ann. CIRP, vol. 41 , no. 1 , p. $577,1992$.

[7] K. Yoshitomi, A. Ishimaru, J. N. Hwang, and J. S. Chen, "Surface roughness determination using spectral correlations of scattered intensities and an artificial neural network technique," IEEE Trans. Antennas Propagat., vol. 41, p. 498, Apr. 1993.

[8] Q. Yang and C. Butler, "An optical fiber sensor for displacement and surface topography measurement using neural networks," in Proc. IMEKO, 1995, p. 1737.

[9] K. Zhang and C. Butler, "A fiber optic sensor for surface roughness and displacement measurement," in Proc. ISMQC'95, 1995, p. 355.

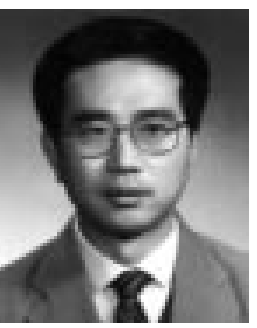

Kuiwei Zhang received the B.Sc. and M.Sc. degrees in mechanical engineering from Northeastern University, Shenyang, China, in 1982 and 1986, respectively. $\mathrm{He}$ is currently pursuing the $\mathrm{Ph} . \mathrm{D}$. degree in the Department of Manufacturing and Engineering Systems, Brunel University, Uxbridge, Middlesex, U.K.

He worked as Assistant Lecturer, Lecturer, and Director of the Tolerance and Measurement Technology Laboratory at Northeastern University. From 1992 to 1993, he was a Visiting Scholar with Brunel Centre for Manufacturing Meteorology, Brunel University. His current research interests are in sensors, measurement, and dimensional meteorology.

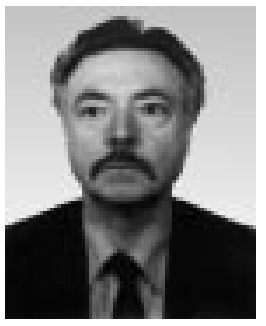

Clive Butler received the B.Sc. degree in physics from the University of Manchester, Manchester, U.K., in 1963, and the M.Sc. and Ph.D. degrees from Imperial College, London, U.K., both in 1968.

He has held a number of industrial posts including Chief Physicist, MOT Ltd., Director, Metronomic Technology Ltd., and Digital Meteorology Ltd. Previously, he was Director of Research at Watford College of Technology, Watford, U.K. Since 1986, he has been a Reader at Brunel University, Uxbridge, Middlesex, U.K. His current research interests are in dimensional meteorology and quality systems. He has presented more than 60 papers in applied optics, printing technology, meteorology, and quality management.

Dr. Butler is a Fellow of the Royal Society of Arts and member of the Institute of Quality Assurance.

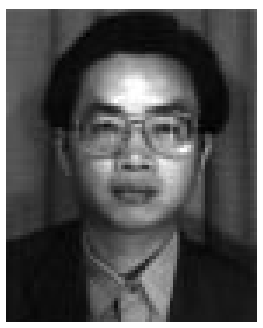

Qingping Yang (M'97) received the Diploma in instrumentation and measurement from Chendu Aeronautical Polytechnic, Chendu, China, in 1983. He was awarded a scholarship to study at Brunel University, Uxbridge, Middlesex, U.K., in 1988, and received the Ph.D. degree in 1992.

He then was an Assistant Engineer, Deputy Head of Department of Measurement and Testing in the Aircraft Structural Strength Research Institute (Ministry of Aerospace), Shaanxi Province, China. He currently lectures on manufacturing meteorology, instrumentation design, and quality control. He has published more than 20 papers on sensors, transducers, instrumentation, and measurement.

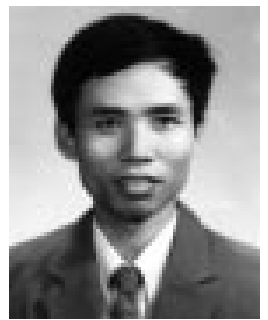

optical fiber sensors.
Yicheng Lu received the B.Sc. and M.Sc. degrees from Northwestern Polytechnical University, Xian, China, in 1981 and 1984, respectively, and the Ph.D. degree from Brunel University, Uxbridge, Middlesex, U.K., in 1992.

He was Assistant Lecturer and Lecturer at Northwestern Polytechnical University. He then joined a research team as a Research Fellow working on optical fiber sensors at Brunel University. He is currently a Senior Engineer at Bookham Technology Ltd., developing integrated optical sensors and 